\title{
Excitation of gap solitons, soliton trains, and soliton sets in finite-sized two-dimensional photonic crystals
}

\author{
Ping Xie and Zhao-Qing Zhang \\ Department of Physics and Institute of Nano Science and Technology (INST), The Hong Kong University of Science \\ and Technology, Clear Water Bay, Hong Kong, China \\ (Received 15 August 2003; published 8 March 2004)
}

\begin{abstract}
We study in detail the excitation of gap solitons in finite-sized two-dimensional photonic crystals under various kinds of source configuration, including two external beams along different incident directions and a point source at different locations inside the sample. We find different types of gap solitons, such as soliton trains along different symmetry axes of the photonic crystal and soliton sets with a higher rotational symmetry. In the case of a single external beam, we find the existence of an optimal beamwidth $d_{\text {opt }}$ for the excitation of gap solitons, and the value of $d_{\text {opt }}$ is close to the diameter of single localized envelopes of the excitation. In the case of a point source, it is found that the excitation threshold depends only on the distance between the source and the nearest cylinder, and its value increases nearly exponentially with distance.
\end{abstract}

DOI: 10.1103/PhysRevE.69.036601

PACS number(s): 42.70.Qs, 42.65.Tg, 63.20.Pw

\section{INTRODUCTION}

Since Yablonovitch [1] and John [2] first proposed that a periodic dielectric structure (photonic crystal) can exhibit a forbidden gap for the propagation of electromagnetic waves, photonic band gap materials have attracted considerable interest both theoretically and experimentally [3]. To employ the high-technology potential of the photonic crystal, it is also crucially important to achieve dynamical tunability of its band gap. One idea of dynamical tunability can be realized by changing the light intensity in so-called nonlinear photonic crystals. Recently, much interest has been focused on the study of nonlinear optical properties of photonic crystals [4].

One of the important phenomena in nonlinear photonic crystals is the existence of nonlinearity-induced selforganized localized states with frequency in the forbidden gaps, which are usually called gap solitons. The term "gap soliton" was first introduced by Chen and Mills in their numerical study of a one-dimensional (1D) Kerr nonlinear superlattice [5]. Later, much interest was focused on the study of gap solitons in 1D superlattices [6]. Recently, John and Akozbek [7] showed the existence of gap solitons in 2D and 3D nonlinear photonic crystals by using coupled-mode theory, which is valid for small dielectric modulations. By using the numerical Green's function, Mingaleev and Kivshar [8] demonstrated the existence of stable gap solitons in a $2 \mathrm{D}$ photonic crystal with a large dielectric contrast. However, it is also of considerable importance to study the excitation of gap solitons in finite-sized 2D photonic crystals using external sources, i.e., the coupling of external sources to the gap soliton. In our recent paper, we demonstrated the excitation of a single gap soliton and soliton trains along the $\Gamma-M$ direction by use of an external slit beam incident upon a finite-sized 2D photonic crystal in a square lattice [9]. In the present work, we study in detail the excitation of gap solitons in finite-sized 2D square photonic crystals using different source configurations. Other types of soliton trains possessing different symmetries have been found. In particu- lar, we find soliton trains along the $\Gamma-X$ direction by using a source with two perpendicular beams along the $\Gamma-M$ direction. These soliton trains are difficult to excite by using a single beam along the $\Gamma-X$ direction. We also find a soliton set with a higher symmetry by using a point source located inside the sample. A soliton set consists of a pair of twosoliton trains attached to each other. In addition, we have studied the properties of gap soliton excitation. In the case of a single external beam, we find that there exists an optimal beamwidth at which the threshold of the incident energy flux is the smallest. The optimal beamwidth is close to the diameter of the localized envelopes of the excitation. In the case of a point source, we find that the threshold of the incident amplitude depends only on the distance between the point source and the nearest cylinder and its threshold value increases nearly exponentially with distance.

\section{MODEL AND CALCULATION METHOD}

We consider a 2D square lattice of dielectric cylinders in air. The radius of the cylinder is $R=0.1 a$, with $a$ the period of the lattice. The dielectric cylinders are Kerr nonlinear, characterized by the usual, weak-field dielectric constant $\varepsilon_{0}$ and the Kerr coefficient $\lambda$. Two finite-sized photonic samples are used in our calculations, which are shown in the insets of Figs. 1(b) and 1(c). The sample in Fig. 1(b) consists of $11 \times 11+11 \times 10=231$ cylinders and that in Fig. $1(\mathrm{c})$ consists of $16 \times 16=256$ cylinders. We assume that the cylinders are parallel to the $z$ axis so that the crystal is characterized by the dielectric constant $\varepsilon(x, y)$. $E$-polarized light, with the frequency $\omega=2 \pi f$, propagating in the $(x, y)$ plane satisfies the following Maxwell equation:

$$
\nabla^{2} E(x, y)+\frac{\omega^{2}}{c^{2}} \varepsilon(x, y) E(x, y)=0
$$

where $E(x, y)$ is the $z$ component of the electric field and $c$ is the speed of light in vacuum. The dielectric constant $\varepsilon(x, y)$ is taken as follows: $\varepsilon(x, y)=\varepsilon_{b}=1$ in air and 


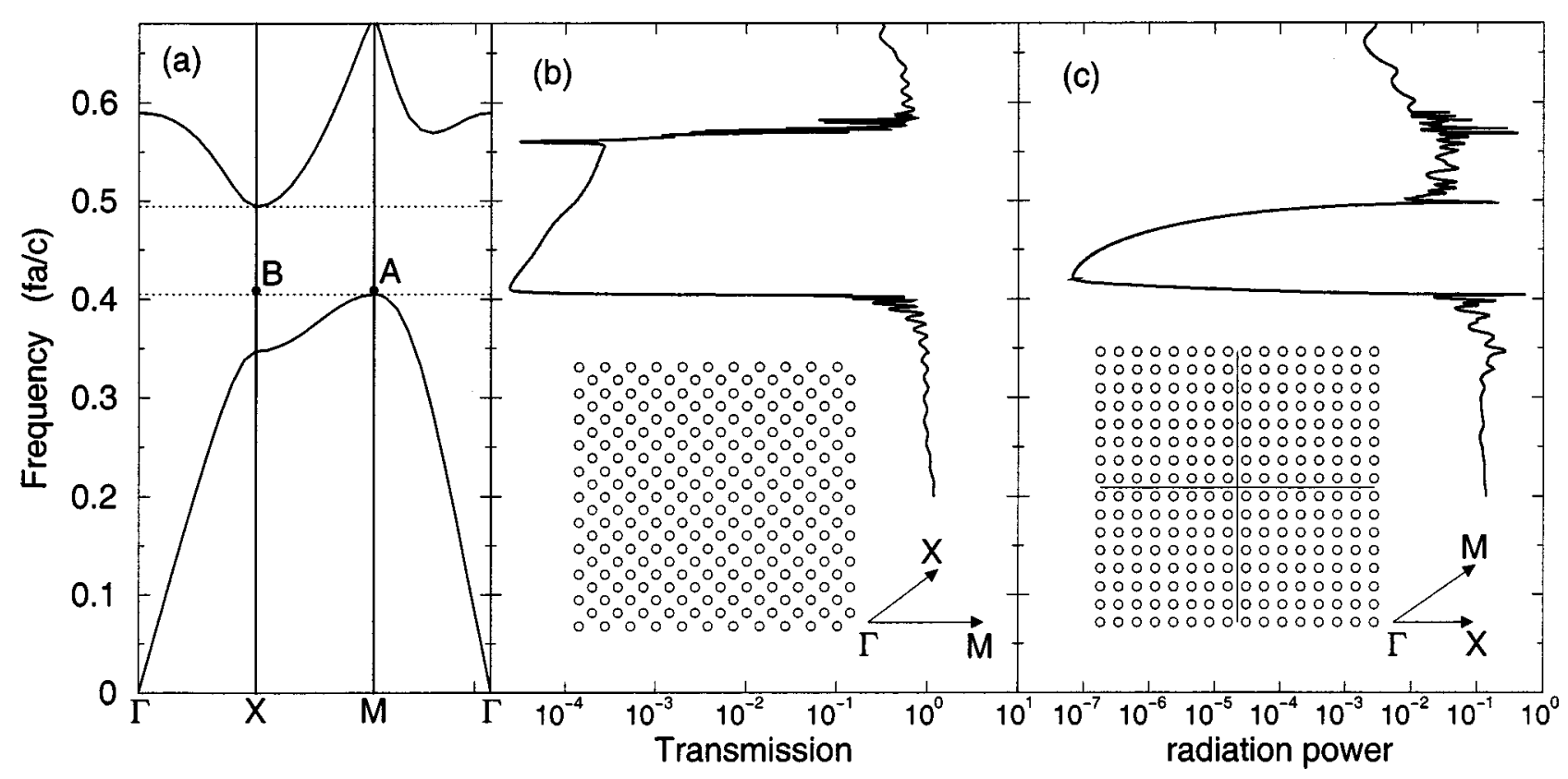

FIG. 1. (a) Band structure of $E$-polarized wave for a square lattice of linear dielectric_cylinders with radius $R=0.1 a$ and dielectric constant $\varepsilon_{0}=13$ in air, where $a$ is the_period of the lattice. (b) Transmission coefficient of a slit beam input from the left face of the linear crystal depicted in the inset. (c) Total radiation power spectrum from a point source inside the linear sample shown in the inset of (b).

$$
\varepsilon(x, y)=\varepsilon_{0}+\lambda|E(x, y)|^{2}
$$

in cylinders.

In this paper, we use the method of multiple scattering $[10,11]$, which is numerically exact, in conjunction with an iterative scheme to solve Eqs. (1) and (2). In performing the multiple-scattering calculation, we divide every cylinder into many cylindrical layers and assume the electric energy in each layer to be constant and equal to the mean intensity in that layer. The validity of this assumption was checked in Ref. [9]. Thus, the dielectric constant in the $n$th cylinder can be written as

$$
\varepsilon_{n m}=\varepsilon_{0}+\lambda\left\langle|E(x, y)|^{2}\right\rangle_{n m},
$$

where the subscript $m$ represents the $m$ th layer of that cylinder and $\left\langle|E(x, y)|^{2}\right\rangle_{n m}$ denotes the mean value of $|E(x, y)|^{2}$ in the $m$ th layer of the $n$th cylinder. The iterative procedure is briefly described as follows. From the value of $E^{(i)}(x, y)$ of the $i$ th step we obtain $\varepsilon_{n m}^{(i+1)}=\varepsilon_{0}+\lambda\left\langle\left|E^{(i)}(x, y)\right|^{2}\right\rangle_{n m}$ from Eq. (3). With this $\varepsilon_{n m}^{(i+1)}$ we obtain $E^{(i+1)}(x, y)$ of the $(i+1)$ th step from the multiple-scattering method $[10,11]$. The above procedure is repeated until the relative value $\left|\varepsilon_{n m}^{(i+1)}-\varepsilon_{n m}^{(i)}\right| / \varepsilon_{n m}^{(i)}$ is smaller than a required value $\delta$ (in this work we take $\left.\delta=10^{-4}\right)$. For the sake of convergence, it is necessary to use $\widetilde{\varepsilon}_{n m}^{(i+1)}=\varepsilon_{n m}^{(i+1)}+\left(\varepsilon_{n m}^{(i+1)}-\varepsilon_{n m}^{(i)}\right) r$ to replace $\varepsilon_{n m}^{(i+1)}$, where $r<1$ is a relaxation factor that should be chosen appropriately in the calculation. In our calculations, we choose $\varepsilon_{0}=13$ and $\lambda=-0.001$, which are usually used in the literature.

In this paper, we use two types of light source to excite the gap solitons. One is the slit source, which is positioned outside the sample, and the other one is a point source, which is positioned inside the sample. For a slit parallel to the $y$ axis with a width $d$ and center position located at $\left(x_{0}, y_{0}\right)$, the electric field in the near field can be expressed as [10]

$$
E(x, y)=a_{0}\left(\frac{k_{b}}{4}\right) \int_{-d / 2}^{d / 2} d y^{\prime}\left(H_{0}\left(k_{b} \rho\right)+i \frac{x-x_{0}}{\rho} H_{1}\left(k_{b} \rho\right)\right),
$$

where $\rho=\sqrt{\left(x-x_{0}\right)^{2}+\left(y-y_{0}-y^{\prime}\right)^{2}}, \quad k_{b}=(\omega / c) \sqrt{\varepsilon_{b}}$, and $a_{0}$ represents the incident amplitude. The function $H_{m}$ with $m=0$ and 1 is the Hankel function of the first kind. For a point source positioned at $\left(x_{0}, y_{0}\right)$, the near-field electric field is given by

$$
E(x, y)=a_{0} H_{0}\left(k_{b} \widetilde{\boldsymbol{\rho}}\right),
$$

where $\widetilde{\rho}=\sqrt{\left(x-x_{0}\right)^{2}+\left(y-y_{0}\right)^{2}}$ and $a_{0}$ represents the incident amplitude.

\section{RESULTS AND DISCUSSION}

First, we give briefly the linear properties of the square photonic crystal considered here. Figure 1(a) shows the band structures for $E$-polarized light. There exists a full gap extending from $\widetilde{f} \equiv f a / c=0.405$ to $\widetilde{f}=0.494$, where $\widetilde{f}$ is the dimensionless frequency. Figure 1(b) shows the transmission spectrum of a slit beam incident upon the crystal shown in the inset of Fig. 1(b) along the $\Gamma-M$ direction from the left face. Here, the transmission coefficient of a slit beam is calculated according to Eq. (11) of Ref. [10]. The transmission spectrum indicates a stop band extending from $\widetilde{f}=0.405$ to 0.569 , which agrees excellently with the position of the partial gap along the $\Gamma-M$ direction shown in Fig. 1(a). In Fig. 1(c) we plot the total radiation power $P_{s}$ in all directions from a point source with the amplitude $a_{0}=1$ located near 

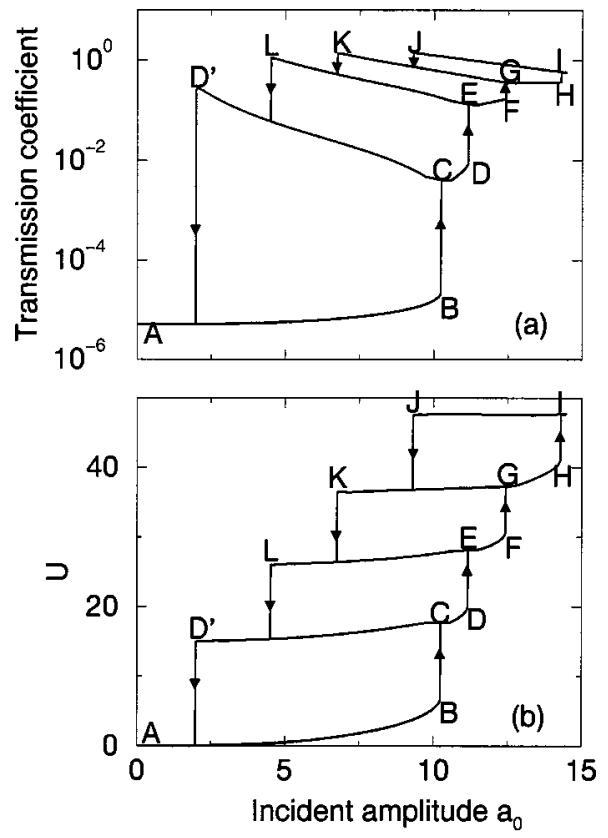

FIG. 2. Transmission coefficient (a) and total energy $U$ accumulated inside the sample (b) as a function of the incident amplitude $a_{0}$ of a slit beam with width $d=2.828 a$ and dimensionless frequency $f a / c=0.407$ in the nonlinear sample shown in the inset of Fig. 1(b). $U$ has the same units as $a_{0}^{2} a^{2}$.

the center of the sample in the inset of Fig. 1(b). The gap position in the radiation power spectrum also agrees well with that of the full gap shown in Fig. 1(a). Below we will present the results for nonlinear photonic crystals for both slit and point source illuminations.

\section{A. Slit source}

Here we are interested in the gap solitons inside the full gap near the lower band edge, i.e., $\widetilde{f}=0.405$ at the $M$ symmetry point, which corresponds to point $A$ in the band structures shown in Fig. 1(a). To excite such gap solitons, it is convenient to use the sample shown in the inset of Fig. 1(b), where the normal of each face is along the $\Gamma-M$ direction.

First, we consider an external slit beam at a fixed frequency incident upon the sample shown in the inset of Fig. 1(b) from the left face. In Fig. 2(a) we show the transmission coefficient as a function of the incident amplitude $a_{0}$ for $\tilde{f}=0.407$. Figure 2(b) is the corresponding total energy accumulated inside the sample, i.e., $U$ $=\frac{1}{2} \int \varepsilon(x, y)|E(x, y)|^{2} d x d y$. When the incident amplitude $a_{0}$ is very small (for example, point $A$ ), the sample is in the linear regime. The beam is in the reflecting state and $U$ is very small. As $a_{0}$ is increased from $A$ to $B$, the beam is still in the highly reflecting state, but $U$ increases continuously. At point $B$ the beam jumps to the transmitting state $C$, and $U$ jumps to a higher value. If we decrease $a_{0}$ from $C$, a maximum of the transmission coefficient occurs at $D^{\prime}$. $U$ changes little along the path $C D^{\prime}$ although the incident amplitude $a_{0}$ decreases a lot. Figure 3(a) shows the intensity distribution at $D^{\prime}$ of the maximum transmission, which cor- (a)
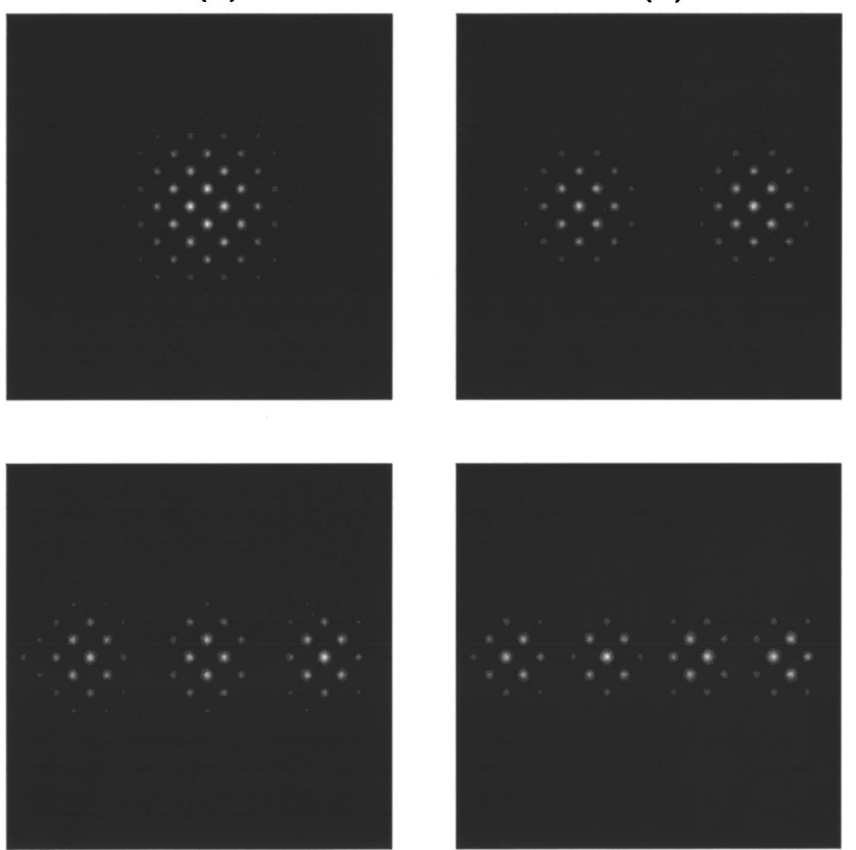

(c)

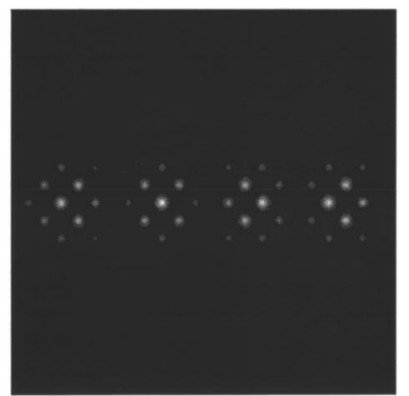

(d)

FIG. 3. Intensity distributions (in arbitrary units) of the singlegap soliton (a) and two- (b), three- (c), and four-soliton (d) trains excited by a slit beam propagating along the $\Gamma-M$ direction or two slit beams oppositely propagating along the $\Gamma-M$ direction in the nonlinear sample shown in the inset of Fig. 1(b).

responds to a single-soliton state. At point $C$ if we do not decrease but continue to increase $a_{0}$, we find that the transmission coefficient increases continuously to $D$ and then jumps suddenly from $D$ to $E$. The corresponding $U$ follows the similar path and jumps to a much higher value at $E$. At point $E$, when we decrease $a_{0}$, we arrive at another maximum of the transmission at $L$. As before, $U$ changes little along the path $E L$. At $E$ if we continue to increase $a_{0}$ another jump from $F$ to $G$ occurs for both the transmission coefficient and $U$. A decrease in $a_{0}$ leads to a maximum of the transmission coefficient at $K$. Points $H, I$, and $J$ are obtained in a similar way. It is seen from Fig. 2(a) that, in some region of the incident amplitude $a_{0}$, multistability of the transmission coefficient occurs in the finite-sized sample. The spatial intensity distributions inside the sample at points $L, K$, and $J$ are shown in Figs. 3(b), 3(c), and 3(d), respectively. They correspond to two-, three-, and four-soliton states, which are called soliton trains and similar to those observed in 1D superlattices [5]. Note that these soliton trains are arranged along the propagating direction of the beam, i.e., along the $\Gamma-M$ direction. If the sample size is enlarged along the $\Gamma-M$ direction, we obtained similar transmission behavior to that shown in Fig. 2 and similar intensity distributions to those shown in Fig. 3. For the case of the single-soliton state shown in Fig. 3(a), we do not find any changes in the spatial size or energy of the excitation, except that the position of the excitation is moved to the center of the enlarged sample. It will be shown later that the excitation of this mode is independent of the source configuration. Thus the localized 
mode in Fig. 3(a) can be considered as an intrinsic excitation of the photonic crystal of an infinite sample. However, this is not true for soliton trains. For instance, in the case of a twosoliton train, the separation of two envelopes is found to increase with sample size. The spatial size of two envelopes is also found to increase with sample size, and tends to saturate to that of the single-soliton state. It will been shown later that two-soliton trains with different symmetries can also be excited in the same sample by using different source configurations. Thus, the soliton trains shown in Figs. 3(b) $-3(d)$ are specific to the sample used in the inset of Fig. 2(b) as well as the source configuration. Unlike the single-soliton states, soliton trains cannot be considered as intrinsic excitations of an infinite photonic crystal. The size of each envelope as well as their relative positions depend on both the source configuration and the sample geometry. In the limit of a large sample, the two envelopes in a two-soliton train are expected to decouple into two independent single-soliton states.

Figure 2 is obtained from a slit beam with width $d$ $=2.828 a$. We see that the beam jumps from a nonpropagating state to a propagating state at the incident amplitude $a_{0}$ $=10.24$ (point $B$ ), which is denoted by $u_{\mathrm{th} 1}$. The second jump of the transmission coefficient occurs at $a_{0}=11.17$ (point $D$ ), which is denoted by $u_{\mathrm{th} 2}$. There are also thresholds $u_{\text {th } 3}=12.44$ (point $F$ ) and $u_{\text {th } 4}=14.29$ (point $H$ ). We emphasize that the solitons and soliton trains shown in Fig. 3 can also be excited with slit beams of other widths, including a plane wave. In Fig. 4(a) we plot $u_{\mathrm{th} i}(i=1,2,3)$ as a function of beamwidth $d$ for $\tilde{f}=0.407$, where $u_{\text {th } 1}$ is shown by the solid curve, $u_{\text {th2 } 2}$ by the dotted curve, and $u_{\text {th } 3}$ by the dashed curve. It is clearly seen that the values of $u_{\text {th } i}$ increase with decreasing $d$. To see the dependence of the total incident energy flux at the thresholds on the beamwidth, in Fig. 4(b) we plot $u_{\mathrm{th} i}^{2} d / 2$, which is proportional to the total incident energy flux, as a function of $d$ for $\widetilde{f}=0.407$. It is interesting to note that there is an optimal beamwidth $d_{\text {opti }}(i=1,2,3)$ at which the total incident energy flux is the lowest. We see that $d_{\text {opt1 }}$ is larger than $d_{\text {opt2 }}$ and $d_{\text {opt2 }}$ is larger than $d_{\text {opt3 }}$. This trend is consistent with the fact that the size of the single localized envelope in Fig. 3(a) is larger than that in Fig. 3(b) and the size of the single localized envelope in Fig. 3(b) is larger than that in Fig. 3(c). Careful observation shows that the values of $d_{\text {opt } i}$ are close to the diameters of the localized envelopes in the corresponding solitons and soliton trains. This assertion is also checked by the calculation of gap solitons at other frequencies. For example, in Fig. 4(c), we plot $u_{\text {th } 1}^{2} d / 2$ as a function of $d$ for $\widetilde{f}=0.406$ (dot-dashed line), $\widetilde{f}$ $=0.407$ (solid line), and $\tilde{f}=0.409$ (long-dashed line). We see that the optimal beamwidth decreases as the frequency is moved away from the gap edge, which is consistent with the fact that the size of the gap soliton is reduced as the frequency is moved into the gap.

Now we consider the case when the sample is illuminated simultaneously by two coherent beams of equal amplitude and equal width. We study the following two cases: (1) The two beams are incident upon the crystal (along the $\Gamma-M$ direction) from the two opposite faces of the sample, e.g., one
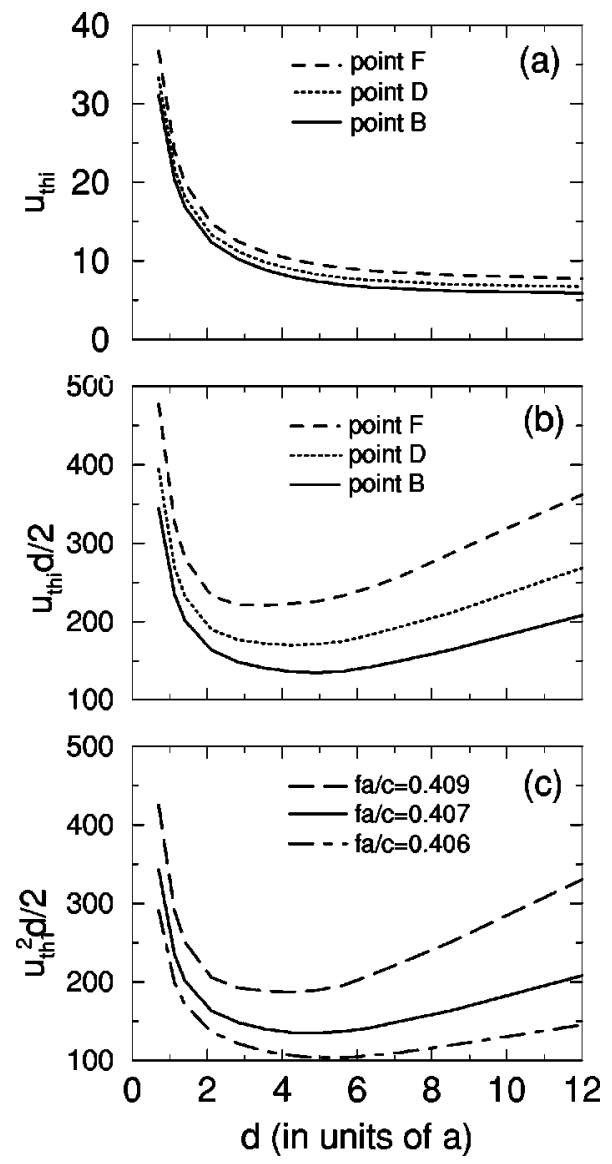

FIG. 4. (a) Thresholds $u_{\text {th1 }}$ (solid line), $u_{\text {th1 }}$ (dotted line), and $u_{\text {th1 }}$ (dashed line) versus $d$ for $f a / c=0.407$. (b) $u_{\text {th } 1}^{2} d / 2$ (solid line), $u_{\mathrm{th} 2}^{2} d / 2$ (dotted line), and $u_{\mathrm{th} 3}^{2} d / 2$ (dashed line) versus $d$ for $\mathrm{fa} / \mathrm{c}$ $=0.407$. (c) $u_{\mathrm{th} 1}^{2} d / 2$ versus $d$ for $f a / c=0.406$ (dot-dashed line), 0.407 (solid line), and 0.409 (long-dashed line). $u_{\text {th } i}$ and $d$ have the same units as $a_{0}$ and $a$, respectively.

from the left and the other from the right; (2) the two beams are perpendicular to each other, while both are along the $\Gamma-M$ direction, with one beam from the left face and the other from the upper face. For the former case, we obtain the same solitons and soliton trains as shown in Fig. 3. The solitons and soliton trains excited by the latter source configuration are shown in Figs. 5(a) and 5(b). We note that, although the position of the single soliton in Fig. 5(a) is different from that in Fig. 3(a), the spatial size and the energy of the gap soliton in Fig. 5(a) are the same as those in Fig. 3(a). It should be pointed out that the single solitons shown in Figs. 3(a) and 5(a) are two equivalent excitations occurring at different locations in a sample. They are related by the translational symmetry of the square lattice. However, the twosoliton train found in Fig. 5(b) is along the $\Gamma-X$ direction, which is different from the two-soliton train shown in Fig. $3(\mathrm{~b})$, where the two envelopes are arranged along the $\Gamma-M$ direction. In this case, they are not related by the rotational symmetry of the square lattice.

The excitation of a two-soliton train along the $\Gamma-X$ direction is particularly interesting. This excitation actually corresponds to point $B$ in the band structures shown in Fig. 1(a). Since this point lies deep inside the partial gap along the $\Gamma-X$ 
(a)

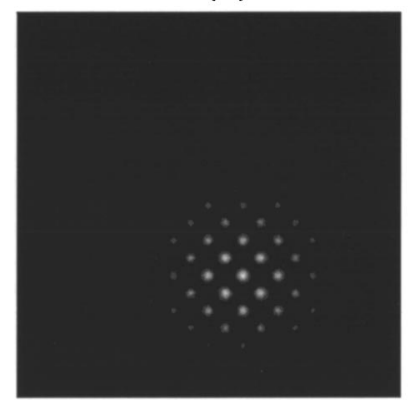

(b)

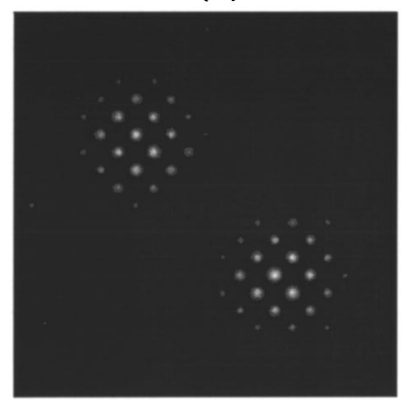

FIG. 5. Intensity distribution (in arbitrary units) of the singlegap soliton (a) and two-soliton train (b) excited by two slit beams perpendicularly propagating along the $\Gamma-M$ direction in the nonlinear sample shown in the inset of Fig. 1(b).

direction, it has a small evanescent decay length for an incident wave along the $\Gamma-X$ direction. Thus, it is rather difficult to excite this soliton train by using a single beam along the $\Gamma-X$ direction. However, it can be easily excited by two perpendicular beams along the $\Gamma-M$ direction as it is much easier for the waves to penetrate into the system along the $\Gamma-M$ direction. It is worth mentioning that the excitation of gap solitons shown in Fig. 5 does not depend on the widths of the two beams.

\section{B. Point source}

The above discussion has been devoted to the case when the nonlinear crystal is excited by the external beam(s). We now turn to excitation by a point source that is positioned inside the nonlinear crystal. As is known, in the linear limit, when the radiation frequency lies inside the band gap there exists no far-field radiation energy from the point source. However, in the nonlinear case, we expect that there can be large radiation power via the formation of gap solitons. For example, in Fig. 6 we plot the total radiation power $P_{s}$ normalized by $a_{0}^{2}$ as a function of the amplitude $a_{0}$ of Eq. (5) at $\tilde{f}=0.407$ for three different positions of the point source inside the crystal shown in the inset of Fig. 1(b), where the dashed curve corresponds to the point source located at $(0,0.2 a)$, the solid curve to $(0,0.4 a)$, and the dotted curve to $(0,0.6 a)$, with the origin $(0,0)$ at the center of the central cylinder of the sample. Each source position gives rise to a hysteresis loop. Its threshold value increases with the distance between the source location and the nearest cylinder of the sample. For the middle one, the normalized radiation power $P_{s} / a_{0}^{2}$ follows the curve $A \rightarrow B \rightarrow C \rightarrow D$ with increasing $a_{0}$. At point $D$, the curve continues according to the path $D \rightarrow E \rightarrow B \rightarrow A$ when $a_{0}$ decreases (see the solid curve). The intensity distribution at point $E$ is shown in Fig. 7(a). It is clear that the excitation at point $E$ where the radiation power is a maximum corresponds to a single gap soliton. This is similar to the point $D^{\prime}$ shown in Fig. 2(a), where the sample is excited using a slit beam. In order to verify that this excitation is intrinsic, i.e., independent of the source configuration, we also examined the intensity profiles at points $F$ and $G$ arising from different point source locations. It is found that, although the positions of the three excitations at points

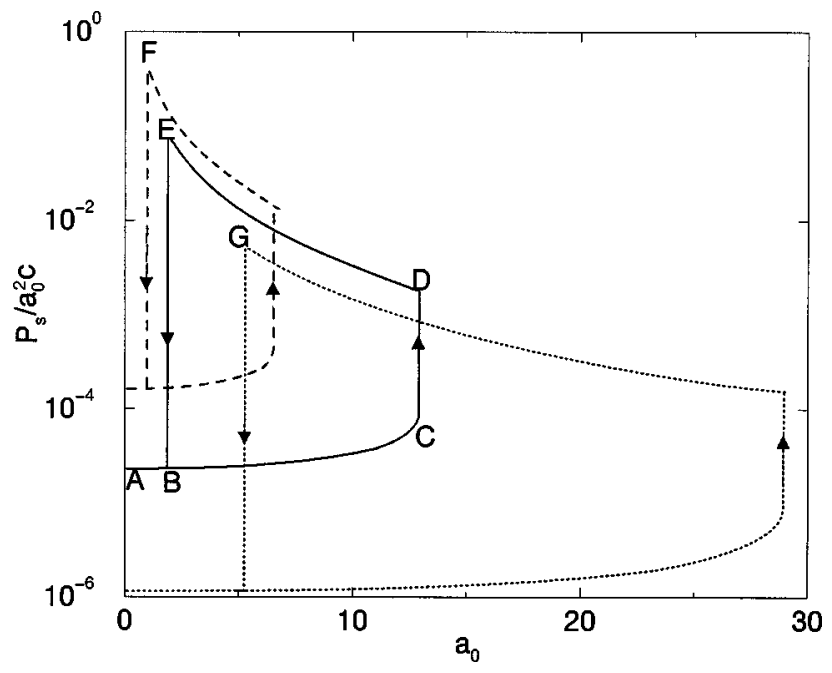

FIG. 6. The total dimensionless radiation power $P_{s} / a_{0}^{2} c$ as a function of the amplitude $a_{0}$ of a point source with $\mathrm{fa} / \mathrm{c}=0.407$ located at $(0,0.2 a)$ (dashed line) (a), $(0,0.4 a)$ (solid line) (b), and $(0,0.6 a)$ (dotted line) $(\mathrm{c})$, where the origin $(0,0)$ is at the center of the central cylinder of the nonlinear sample shown in the inset of Fig. 1(b). The radiation power $P_{s}$ is the integrated far-field energy flux defined in Ref. [10].

$E, F$, and $G$ are different, the spatial sizes and the energies of the three excitations are the same. Furthermore, they also have the same values as those shown in Figs. 3(a) and 5(a) obtained by using outside illumination.

As we pointed out before in Fig. 6, the threshold of the incident amplitude $a_{0}$ (e.g., point $C$ ) at which the system jumps from a nonradiating state to a radiating state depends sensitively on the location of the point source. Considerable calculations show that this threshold $a_{\text {th }}$ is dependent only on the distance $l$ between the point source and the nearest cylinder lying near the center of the sample and is independent of the orientation of the source position relative to the nearest cylinder. In Fig. 8 we plot the threshold as a function of this distance $l$ on a semilogarithmic scale. It is seen that the threshold value increases nearly exponentially with the distance $l$. This result is related to the evanescent nature of the (a)

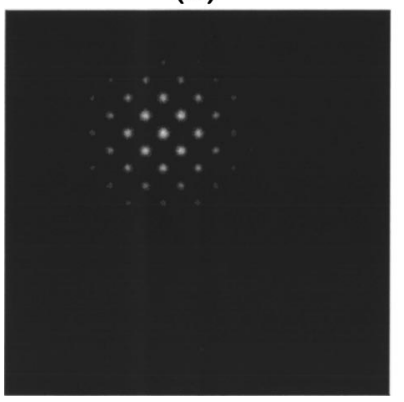

(b)

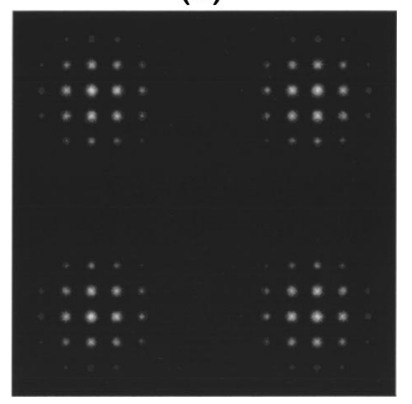

FIG. 7. (a) Intensity distribution (in arbitrary units) of the single-gap soliton excited by a point source located inside the nonlinear sample shown in the inset of Fig. 1(b). (b) Intensity distributions (in arbitrary units) of the four-soliton set excited by a point source located at the center of the nonlinear sample shown in the inset of Fig. 1(c). 


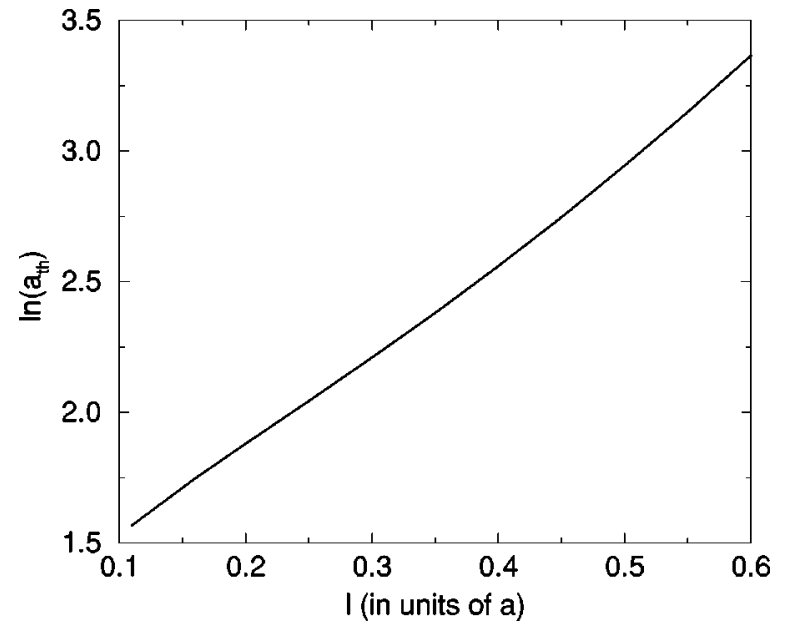

FIG. 8. The logarithm of the threshold of the point source amplitude $a_{0}$ as a function of the distance $l$ from the point source to its nearest cylinder which is located near the center of the nonlinear sample shown in the inset of Fig. 1(b).

waves inside the gap. In addition to the single gap soliton shown in Fig. 7(a), we have also seen the excitation of twoand three-soliton trains by using a point source. For example, this can be achieved by increasing the incident amplitude $a_{0}$ at point $D$ in Fig. 6, similar to the multistability curve shown in Fig. 2. However, in the most general case, the gap soliton trains we have observed are along the $\Gamma-M$ direction, i.e., the ones shown in Figs. 3(b) and 3(c).

In order to excite soliton trains along the $\Gamma-X$ direction, we have to choose a symmetric sample such as the one shown in the inset of Fig. 1(c). The point source is located on two perpendicular symmetry lines of the sample (solid lines), but not at the center of the sample where the two solid lines meet. In this case, we are able to excite a two-soliton train along the $\Gamma-X$ direction, similar to the one shown in Fig. 5(b). If we put the point source at the center of the sample where the two solid lines meet, we also observe a different type of gap soliton, which is shown in Fig. 7(b). This gap soliton consists of four localized envelopes and can be called a four-soliton set. Compared to gap soliton trains, a soliton set has a higher rotational symmetry. It can be considered as a pair of two-soliton trains closely attached to each other.

Finally, we give an estimate of the source power required to excite the gap solitons presented in this paper. The Kerr coefficient $\lambda$ is related to the third nonlinear susceptibility $\chi^{(3)}$ by the equation $\lambda=12 \pi \chi^{(3)}$ [12]. Thus from $\lambda=-0.001$ we have $\chi^{(3)} \approx-2.65 \times 10^{-5}$ esu if the units of optical electric field are chosen as statvolt $/ \mathrm{cm}$. The magnitude of $\chi^{(3)}$ so chosen is smaller than that of GaAs $\left(\sim 6.5 \times 10^{-4}\right.$ esu [12], p. 163). Therefore, for this value of $\chi^{(3)}$ the incident electricfield amplitude, e.g., $a_{0}=10$ shown in Fig. 2, corresponds to a laser power of $I=\frac{1}{2} c \varepsilon_{0}\left|E_{0}\right|^{2} \approx 18 \mathrm{~kW} / \mathrm{cm}^{2}$, where $\left|E_{0}\right|$ $=a_{0}=10 \mathrm{stat}$ olt $/ \mathrm{cm}$.

\section{SUMMARY}

We studied the excitation of gap solitons, soliton trains, and soliton sets in finite-sized 2D photonic crystals in a square lattice by using two types of light source-slit and point sources. For the slit source we studied three cases. The first case is that a beam is incident upon the crystal along the $\Gamma-M$ direction. The second case is that two beams are incident upon two opposite faces of the crystal along the $\Gamma-M$ direction. The third case is that the two beams are incident upon two adjacent faces of the crystal along the $\Gamma-M$ direction. For the first and second cases, we obtained exactly the same soliton and soliton trains, which are arranged along the $\Gamma-M$ direction. For the third case, we obtained a singlesoliton state that is related to the single-soliton state obtained from the first two cases by translational symmetry. We also obtained a two-soliton train along the $\Gamma-X$ direction that is different from the two-soliton train along the $\Gamma-M$ direction obtained in the first and second cases. They cannot be related by the rotational symmetry of the system. In the case of single-beam incidence, we found the existence of an optimal beamwidth at which the threshold of the incident energy flux is the smallest. The optimal beamwidth is close to the diameter of the localized envelope of the single soliton or the localized envelope of the soliton trains.

For the case of a point source, we observed the excitation of single solitons and soliton trains along the $\Gamma-M$ directions. The threshold of the incident amplitude needed to excite the gap soliton is sensitively dependent on the position of the point source, and it is dependent only approximately on the distance from the point source to the nearest cylinder. The threshold increases nearly exponentially with the distance. We can also excite the soliton trains along the $\Gamma-X$ direction if a symmetrical sample is chosen and the point source is located along the two symmetry axes of the sample. When the point source is located at the center of the sample, a different type of gap soliton can be excited. This soliton consists of a pair of closely attached two-soliton trains.

\section{ACKNOWLEDGMENT}

This work was supported by Hong Kong Research Grants Council Grant No. CA02/03/SC01.
[1] E. Yablonovitch, Phys. Rev. Lett. 58, 2059 (1987).

[2] S. John, Phys. Rev. Lett. 58, 2486 (1987).

[3] See, for example, Photonic Band Gap Materials, edited by C. M. Soukoulis (Kluwer Academic, Dordrecht, 1996); J. D. Joannopoulos, R. D. Meade, and J. N. Winn, Photonic Crystals (Princeton University Press, Princeton, NJ, 1995); K. Sakoda,
Optical Properties of Photonic Crystals (Springer-Verlag, Berlin, 2001).

[4] See, for example, M. Scalora, J. P. Dowling, C. M. Bowden, and M. J. Bloemer, Phys. Rev. Lett. 73, 1368 (1994); P. Tran, Opt. Lett. 21, 1138 (1996); E. Centeno and D. Felbacq, Phys. Rev. B 62, R7683 (2000); C. M. Bowden and A. M. Zheltikov, 
J. Opt. Soc. Am. B 19, 2046 (2002), and references therein. [5] W. Chen and D. L. Mills, Phys. Rev. Lett. 58, 160 (1987).

[6] D. L. Mills and S. E. Trullinger, Phys. Rev. B 36, 947 (1987); D. N. Christodoulides and R. I. Joseph, Phys. Rev. Lett. 62, 1746 (1989); C. M. de Sterke and J. E. Sipe, in Progress in Optics XXXIII, edited by E. Wolf (Elsevier, Amsterdam, 1994), Chap. III; S. Lee and S.-T. Ho, Opt. Lett. 18, 962 (1993); N. G. R. Broderick, D. Taverner, and D. J. Richardson, Opt. Express 3, 447 (1998).

[7] S. John and N. Akozbek, Phys. Rev. Lett. 71, 1168 (1993); N.
Akozbek and S. John, Phys. Rev. E 57, 2287 (1998).

[8] S. F. Mingaleev and Y. S. Kivshar, Phys. Rev. Lett. 86, 5474 (2001).

[9] P. Xie, Z. Q. Zhang, and X. Zhang, Phys. Rev. E 67, 026607 (2003).

[10] L. M. Li and Z. Q. Zhang, Phys. Rev. B 58, 9587 (1998).

[11] G. Tayeb and D. Maystre, J. Opt. Soc. Am. A 14, 3323 (1997).

[12] See, for example, R. W. Boyd, Nonlinear Optics (Academic, New York, 1992), Chap. 4.1, p. 160. 